\title{
Pseudoangiomatous Nodular Stromal Hyperplasia of the Breast (PASH): Case Report and Review of the Literature
}

\author{
Sounni $\mathrm{A}^{1^{*}}$, Jayi $\mathrm{S}^{2}$, Fdili A FZ ${ }^{3}$, Chaara $\mathrm{H}^{4}$, Melhouf MY A
}

${ }^{1-5}$ Department of Obstetric Gynecology Ii Chu Hassan Ii Fes, Sidi Mohamed Ben Abdellah University Faculty of Medicine and Pharmacy Morocco

\author{
DOI: $10.36348 /$ sijog.2021.v04i02.004
}

| Received: 09.02.2021 | Accepted: 22.02.2021 | Published: 27.02.2021

*Corresponding author: Sounni A

\section{Abstract}

Pseudoangiomatous nodular stromal hyperplasia of the breast (PASH) is a benign lesion characterized by the presence of anastomotic clefts lined with flattened cells giving a pseudo-vascular appearance [1], it is a rare pathological entity that manifests itself most often in perimenopausal patients. It poses a differential diagnostic problem with fibroadenoma, phyllodes tumor or hamartoma and it is the histology that confirms the diagnosis. We describe through a series of studies and also through our case the clinical, histological and therapeutic particularity of these tumors.

Keywords: Pseudoangiomatous stromal hyperplasia, cancer, treatment.

Copyright (C) 2021 The Author(s): This is an open-access article distributed under the terms of the Creative Commons Attribution 4.0 International
License (CC BY-NC 4.0) which permits unrestricted use, distribution, and reproduction in any medium for non-commercial use provided the original
author and source are credited.

\section{INTRODUCTION}

Pseudoangiomatous stromal hyperplasia (PASH) is a rare pathological entity which is characterized histologically by an impairment simulating a vascular lesion. It was first described by Vuitch in 1986 [2]. It manifests itself most often by one or more nodules or in the form of an enormous mass responsible for breast asymmetry, but rarely in the form of a subclinical radiological lesion [2]. Imaging does not make it possible to make the diagnosis because of several differential diagnoses including a fibroadenoma or phyllodes tumor; moreover, a biopsy makes it possible to make the diagnosis. The treatment is based on a complete excision of the tumor.

\section{CLINICAL CASE}

A 19-year-old single patient with no notable pathological history. She was presented with a lump in the right breast, which has rapidly increased in size over 6 months. The senological examination objectified B Cup breasts with a mass of $10 \mathrm{~cm}$ by $10 \mathrm{~cm}$ occupying the entire upper external quadrant, mobile in relation to both skin and muscle planes, of firm and very limited consistency. Breast ultrasound revealed a hypoechoic, trilobed, well-defined lesion, occupying the entire upper external quadrant, measuring $128 * 70 \mathrm{~mm}$ classified ACR3.
The patient underwent a large lumpectomy by enucleation (Figure 1.2) in which the pathological examination of the surgical specimen revealed lesions of pseudoangiomatous stromal hyperplasia (Figure 3).

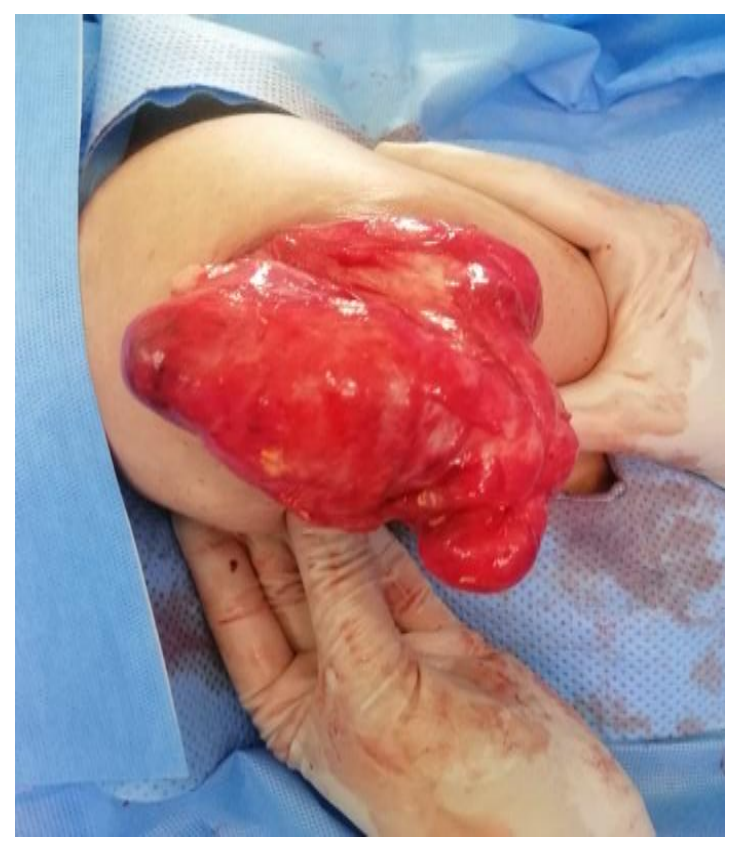

Fig-1 


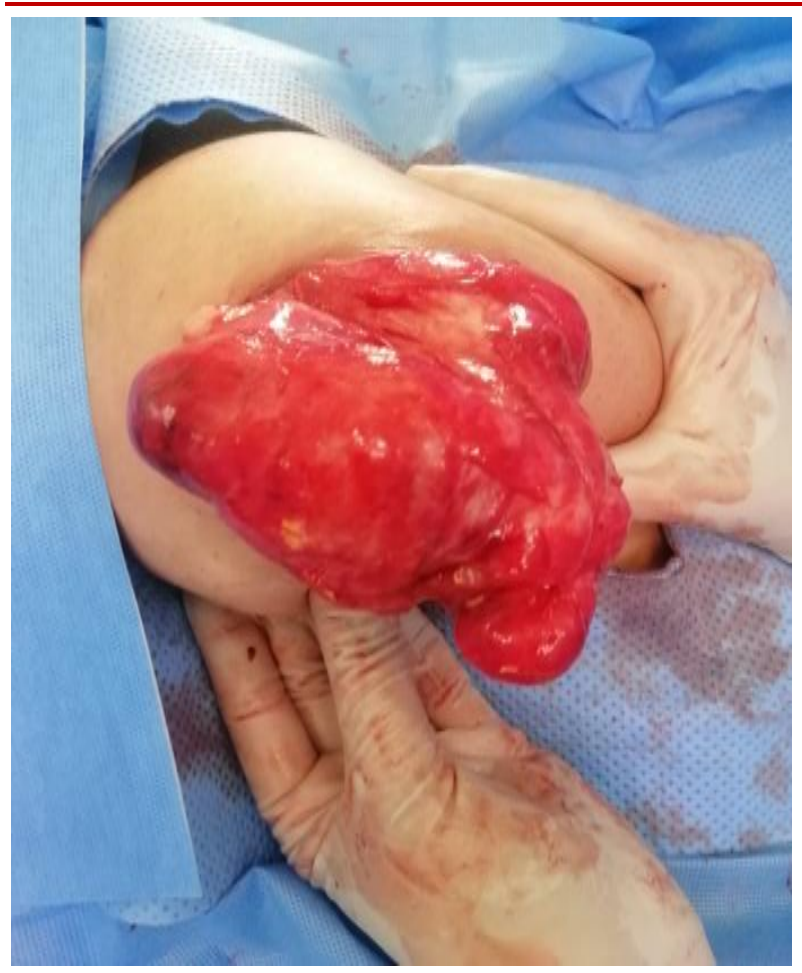

Fig-2

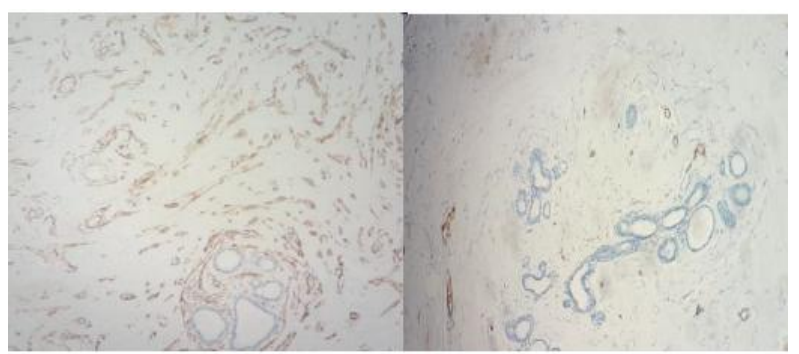

Fig-3: Histological appearance of PASH

\section{DISCUSSION}

Pseudoangiomatous stromal hyperplasia is a benign parenchymal lesion characterized by anastomotic clefts mimicking vascular lesion [1]. It is a common pathological entity in perimenopausal women $[2,3]$, but it can be seen at any age from 14 to 67 years old as is the case with our patient who is 19 years old.

Epidemiologically, it is a rare mammary tumor. Only 250 cases that have been reported up to 2014 through series with a limited number, or through isolated clinical cases (Canlobre 2015).

A series of 9056 surgical biopsies were performed by Degnim of which only 579 returned in favor of PASH (6.4\%) [4]. In our context, only two cases have been discovered since 2016. The pathogenesis remains unknown currently. Some researchers suggest that it is a response of myofibroblasts to several hormonal stimuli, especially progesterone. Therefore, this lesion will be a response from a tissue previously impregnated with estrogen since these tumors strongly express hormone receptors
[5]. Additionally, it is a lesion which does not present a progressive profile towards neoplasia and it has been rarely associated with precancerous lesions, especially atypical hyperplasia with a risk of at least $5 \%$ of being associated with these precursors [1].

The clinical examination generally finds a nodule or a mass of a firm consistency; it is mobile in relation to both skin and muscle planes and unilateral and rarely bilateral. The average size is $4 \mathrm{~cm}$ (between 2 and $10 \mathrm{~cm}$ ). It is painless with rapid growth [5], which was objectified in our patient. Imaging has no specific radiological characteristics; mammography often shows a homogeneous oval or round opacity with clear and regular contours and without intratumoral calcification or architectural disorganization of the adjacent tissue which can lead to a malignant lesion [5]. The breast ultrasound shows a hypoechoic bilobed or multilobed lesion with clear contours and inconsistently lateral shadow and / or posterior reinforcement.

The radiological size varies between 0.9 and $10 \mathrm{~cm}$ (mean at $4 \mathrm{~cm}$ ) superimposable on the clinical data $[2,5]$. This was typically on the breast ultrasound of our patient with a large lobulated lesion and regular seams. The clinical examination and imaging do not make it possible to diagnose. The diagnosis is based on the anatomopathological study of the breast biopsy, which makes it possible to highlight a predominant fibrous stroma with a vast network of anastomosed slits lined with spindle cells. Evoking vascular cells this aspect is characteristic of pseudoangiomatous stromal hyperplasia (PASH) lesions [2-5]. Several differential diagnoses arise with these lesions, especially low-grade angiosarcoma which is a hyper malignant tumor. Vascularized (multiple vascular lumens containing blood and completely surrounded by endothelial cells), myofibroblastoma or phyllodes tumor [2].

Currently, therapeutic management is not coded for simple clinical monitoring, medical treatment or surgery. According to retrospective studies including 40 patients, the evolution was marked by stability in $80 \%$ of cases. A gradual increase in height was noted in $20 \%$ of cases $[1,6]$.

The place of medical treatment with tamoxifen has not proven its effectiveness in young patients or in men with gynecomastia. It has found its place in cases of diffuse involvement $[1,2]$.

Surgery is currently the most effective therapeutic means. There are currently no data or recommendations concerning the limits of resection given the exceptional nature of recurrence. The majority of researchers recommend healthy limits. Several surgical treatment options depending on the sizeof the tumor and breast volume, which are either a large lumpectomy with or without oncoplasty, or a mastectomy if the tumor is very large and the cosmetic 
results are unsatisfactory after conservative treatment [2].

It is a lesion that does not have a progressive profile towards neoplasia and it has been rarely associated with precancerous lesions, including atypical hyperplasia with a risk of at least $5 \%$ of being associated with these precursors [1].

According to the majority of researchers, no monitoring schedule has been recommended due to the exceptional nature of these tumors of simple monitoring at 6 months or after 4 years $[1,2]$.

\section{CONCLUSION}

PASH is a rare benign mesenchymal tumor in premenopausal women whose diagnosis with certainty is made by biopsy. The treatment is generally based on surgical excision whose evolution is often favorable.

\section{REFERENCE}

1. Management of epidemiologically rare benign breast tumors. (2015). Journal of Obstetric Gynecology and Reproductive Biology, 12-13-14.
2. Pseudo-angiomatous stromal hyperplasia of the breast: About a case. (2018). Ernest Barekensabe, Imane Gouzi, IOSR Journal of Nursing and Health Science, December, 8, 2-3

3. Pseudoangiomatous Stromal Hyperplasia (PASH) of the Breast. (2020). An Uncommon Finding in an Uncommon Patient, Alessandra Surace, Viola Liberale, American journal of case reports, Am J Case Rep, 21: e919856.

4. Pseudoangiomatous Stromal Hyperplasia and Breast Cancer Risk. (2010). Annals of surgical oncology, 12-17.

5. Pseudoangiomatous nodular stromal hyperplasia of the breast. (2005). An anatomo-radio-clinical entity to be rediscovered, Anne Heckly 1, Martine Clouet 2, Florence Burtin 3, Patrick Tas 3, Jean Levêque 1, imaging of women. $140-145$.

6. Pseudoangiomatous stromal hyperplasia tumor. (2008). A clinical, radiologic and pathologic study of 26 cases, Marco Ferreira1, Constance T Albarracin2 and Erika Resetkova2, Modern Pathology, 21, 201-207. 